\title{
PSICOLOGIA NA ATENÇÃO PRIMÁRIA À SAÚDE: DESENHANDO POSSÍVEIS CENÁRIOS DE ATUAÇÃO A PARTIR DA FORMAÇÃO
}

PSYCHOLOGY IN PRIMARY HEALTH CARE: DRAWING POSSIBLE SCENARIOS OF ACTION BASED ON TRAINING

PSiCOLOGÍA EN LA ATENCIÓN PRIMARIA A LA SALUd: DISEÑANDO POSIBLES ESCENARIOS DE ACTUACIÓN A PARTIR DE LA FORMACIÓN

\author{
Ana Ester Maria Melo Moreira \\ Brenda Brito Ramos 2 \\ Thalia Ariadne Peña Aragão ${ }^{3}$ \\ Letícia Dias Baroni 4 \\ Kerolayne Castro Fontenele ${ }^{5}$ \\ José Lucas Soares Araújo 6
} Psicologia; Saúde Pública; Atenção Primária à Saúde.

Descriptors: Psychology; Public Health; Primary Health Care.

Descriptores: Psicología; Salud Pública; Atención Primaria a la Salud.

Submetido: $01 / 06 / 2020$

Aprovado:

$16 / 10 / 2021$

Autor(a) para Correspondência: Ana Ester Maria Melo Moreira Universidade Federal do Piauí Endereço: Avenida São Sebastião, 2819, Nossa Senhora de Fátima CEP: 64202-020

E-mail: estermelouniversidade@ gmail.com

\section{RESUMO}

A Psicologia na Atenção Primária à Saúde (APS) é um campo recente de estudos e práticas. 0 objetivo deste artigo é apresentar um relato de experiência do estágio profissional em Psicologia na Atenção Primária à Saúde no âmbito do Sistema Único de Saúde (SUS). Compreende-se que o processo de inserção da Psicologia no SUS abrigou como marcador ideológico o movimento da Reforma Psiquiátrica. Considerando a atuação da Psicologia na APS, o processo de institucionalização ocorre com a implantação do Núcleo de Apoio à Estratégia Saúde da Família. Diversos movimentos da academia, dos sistemas de saúde e dos movimentos sociais mobilizaram esforços para pensar a atuação. A metodologia deste relato de experiência tem como foco a observação participante. A estratégia de coleta de dados envolveu o uso do diário de campo. o processo de análise se deu por meio da sistematização do diário de campo. Com a análise e a discussão dos resultados, emergiram os seguintes cenários de atuação: a) territorialização; b) clínica ampliada; c) abordagens comunitárias; d) educação permanente em saúde; e e) fortalecimento da participação e do controle social. Por fim, aponta-se que esse cenário de prática se mostra ameaçado pelas recentes mudanças nas políticas públicas de saúde.

\footnotetext{
1. Psicóloga. Doutoranda de Saúde Coletiva. Universidade Federal do Piauí. E-mail: estermelouniversidade@gmail. com ORCID: https://orcid.org/0000-0002-8313-4375

2. Psicóloga. Residente do Programa de Residência Multiprofissional de Saúde da Família. Escola de Saúde Pública Visconde de Saboia.E-mail: brendabritoramoss@gmail.com ORCID: https://orcid.org/0000-0002-4646-4826 3. Psicóloga. Residente do Programa de Residência Multiprofissional em Saúde Coletiva. Instituto Aggeu Magalhães/ Fiocruz-PE. E-mail: thaliariadne.pena@gmail.com ORCID: https://orcid.org/0000-0001-6370-5612 4. Psicóloga. Especialista em Saúde da Família e Comunidade. Universidade Federal Delta do Parnaíba. E-mail: alegria.baroni@gmail.com 0RCID: https://orcid.org/0000-0002-5825-1385

5. Psicóloga. Centro de Atenção Psicossocial da Secretaria Municipal de Saúde. Prefeitura Municipal de PiracurucaPI. E-mail: kerolayne.amaral@hotmail.com 0RCID: https://orcid.org/0000-0003-3387-6327

6. Psicólogo. Mestrando do Programa de Pós-graduação em Psicologia. Universidade Federal do Delta do Parnaíba. E-mail: joselucas2126@gmail.com ORCID: https://orcid.org/0000-0002-4541-9329
} 


\section{ABSTRACT}

Psychology in Primary Health Care (PHC) is a recent field of studies and practices. This article aims to present an experience report of the professional internship in Psychology in Primary Health Care in the scope of the Brazilian Unified Health System (Sistema Único de Saúde, SUS). We understand that the insertion process of Psychology in the SUS sheltered the Psychiatric Reform movement as an ideological marker. Considering the role of Psychology in PHC, the institutionalization process occurs with the implementation of the support center for the Family Health Strategy. Several movements in academia, health systems, and social movements mobilized efforts to think about this action. The methodology of this experience report is focused on participant observation. The data collection strategy involved the use of the field diary. The analysis process took place by means of the systematization of the field diary. With the analysis and discussion of the results, the following scenarios emerged: a) territorialization; b) expanded clinic; c) community approaches; d) permanent health education; and e) strengthening of participation and social control. Finally, we pointed out that this practice scenario is threatened by recent changes in public health policies.

\section{RESUMEN}

La psicología en la Atención Primaria a la Salud (APS) es un campo reciente de estudios y prácticas. El objetivo del artículo es presentar relato de experiencia de la pasantía profesional en Psicología en la Atención Primaria a la Salud en el ámbito del Sistema Único de Salud. Se comprende que el proceso de inserción de la Psicología en SUS abrigó como marcador ideológico el movimiento de la Reforma Psiquiátrica. Considerando la actuación de Psicología en APS, el proceso de institucionalización ocurre con implementación del núcleo de apoyo a la Estrategia Salud de la Familia. Diversos movimientos de la academia, los sistemas de salud y los movimientos sociales movilizaron esfuerzos para pensar la actuación. La metodología del relato de experiencia tiene como foco observación participante. La estrategia de coleta de datos involucró el uso del diario de campo. El proceso de análisis se dio por la sistematización del diario de campo. Con el análisis y la discusión de los resultados, emergieron los siguientes escenarios de actuación: a) territorialización; b) clínica ampliada; c) abordajes comunitarios; d) educación permanente en salud; y e) fortalecimiento de participación y control social. Por fin, se apunta que ese escenario de práctica se muestra amenazado por recientes cambios en las políticas públicas de salud.

\section{INTRODUÇÃO}

0 processo de organização da inserção da Psicologia na Saúde Coletiva é recente. Seu marco inicial consistiu na Reforma Sanitária, na Reforma Psiquiátrica e na criação do Sistema Único de Saúde (SUS). 0 processo de inserção da Psicologia na Atenção Primária à Saúde (APS) ocorreu especificamente a partir desses movimentos históricos de ampliação da luta pelo direito à saúde e tem como matriz jurídico-normativa a portaria de implantação e regulamentação do Núcleo de Apoio à Saúde da Família (NASF) ${ }^{1}$. É importante relembrar que o processo de inserção histórica da Psicologia na Atenção Básica à Saúde $(A B S)^{1-5}$ tem na Reforma Psiquiátrica ${ }^{2}$ seu marcador mais relevante, sendo consensual na literatura a relevância desse movimento como sujeito político que tensiona o lugar do manicômio na atenção à saúde mental e na reorganização do modelo de atenção à saúde mental.

A partir desse momento, teve início a organização da atuação da psicologia nas políticas públicas de saúde e na abertura de campos de atuação profissional com a criação do SUS. A Reforma Psiquiátrica² foi um processo crítico à psiquiatria tradicional e à organização da atenção à saúde mental centrada na estrutura manicomial, apontando a necessidade de organização desse modelo de atenção à saúde mental voltada à ampliação das práticas clínicas, ao desenvolvimento de abordagens comunitárias e à criação de serviços substitutivos de cuidado em saúde mental, nos quais o sujeito em situação de sofrimento fosse protagonista de seu processo terapêutico integrado à família e ao território ${ }^{6}$. A atuação se fundamenta na clínica ampliada, partindo dos diversos territórios existenciais do sujeito e da sua vida social.

Diversos autores, na literatura mais recente, situam a atuação da Psicologia na Saúde Coletiva $a^{2,4,5,7,8}$ contrapondo o lugar histórico do psicólogo atrelado a concepções de atuação marcadas pelas dimensões biomédica, manicomial e por pressupostos clínicos 
tradicionais da saúde pública ${ }^{9}$. 0 psicólogo se situa nesse campo ético-político, uma vez que compreende a atuação da Psicologia na Saúde Coletiva e deve desenvolver processos de trabalho na organização de sistemas e serviços de saúde, nas práticas de atenção à saúde e no campo das práticas sociais ${ }^{9}$. Considerando tal processo, o psicólogo atua orientado por processos de territorialização, clínica ampliada, por abordagens comunitárias, pelo apoio institucional, pela educação permanente em saúde e pelo fortalecimento da participação popular nos sistemas e serviços de saúde, com ênfase no sujeito e no território. Essas dimensões estão em íntima sintonia com a APS 8 , $10-12$ como categoria teórica e campo de práticas proposto pelo movimento da Reforma Sanitária, apontando a importância da APS ampliada, que trabalha com a abordagem no território e com a determinação social da saúde, resistindo aos modelos das agências multilaterais de APS seletiva, centrados nas doenças mais prevalentes das populações em situação de pobreza, com foco em programas específicos de saúde. Dessa forma, a atuação da Psicologia na APS busca superar a concepção tradicional de clínica, dialogando com os pressupostos teóricos acima delimitados.

É nesse contexto que emerge a justificativa deste relato de experiência: a importância da articulação teórico-prática entre Psicologia e o campo da Saúde Coletiva com ênfase na APS; de fortalecer teoricamente as experiências de saúde mental na APS; de sistematizar experiências neste campo como dispositivo da Reforma Psiquiátrica Brasileira; e, finalmente, a relevância se fundamenta no desafio ético-político de fortalecimento teórico do campo em um momento de forte recuo das políticas de saúde no que tange à integralidade na ABS.

Dessa forma, a partir da produção científica na área, do levantamento da produção teórica da área de Psicologia na APS 3 -8,11-14, dos documentos técnicos do Conselho Federal de Psicologia (CFP) e da base jurídico-normativa do Ministério da Saúde (MS), que apontam a atuação da Psicologia na APS, desenvolveuse uma proposta de atuação para o estágio profissional que constitui o objeto de discussão deste artigo. A proposta foi desenvolvida no Estágio Profissional do curso de Psicologia da Universidade Federal do Delta do Parnaíba (UFDPAR), com avaliação participativa da comunidade de aprendizagem desenvolvida por universidade, sistema de saúde e comunidade, isto é, docentes, discentes, trabalhadores da Estratégia Saúde da Família e movimentos comunitários. A avaliação foi sistematizada pelos relatórios finais do Estágio Profissional, apontando a importância da sistematização desta experiência.

Assim, o objetivo deste trabalho é relatar a experiência de atuação da Psicologia na Atenção Primária à Saúde.

\section{METODOLOGIA}

0 presente artigo é o relato de experiência da atuação da Psicologia na Atenção Básica à Saúde desenvolvida no Estágio Profissional I Ênfase Saúde Coletiva, na UFDPAR, dialogando com a Clínica e a Saúde Coletiva ${ }^{15,16}$ como dispositivos de educação permanente em saúde ${ }^{17}$. A metodologia deste relato de experiência trabalha com os pressupostos das metodologias participativas de intervenção $0^{13,14,18,19}$, que objetivam desenvolver um processo de encontro entre a academia, os serviços de saúde e a comunidade. As metodologias participativas ${ }^{13,14,18}$ compreendem que todos os sujeitos inseridos na vivência são atores na produção de conhecimento e buscam superar o distanciamento entre saber popular e saber científico, ou seja, entre universidade, profissionais da saúde e sociedade. Dessa forma, utilizou-se a técnica da observação participante ${ }^{17-19}$ com o objetivo de compreender e sistematizar todo o processo de inserção na realidade social.

Considerando tais pressupostos, o delineamento deste relato de experiência adotou a técnica do diário de campo ${ }^{17-19}$, buscando sistematizar as dimensões objetivas, reflexivas e analíticas da vivência, além de construir uma narrativa na terceira pessoa do plural. Transversalmente, sistematizou todas as vivências no espaço de formação, inserção e atuação do estágio profissional de Psicologia na APS. A experiência ocorreu no período de 2015 a 2020 por meio da disciplina de Estágio Profissional I Ênfase Saúde Coletiva, da UFDPAR.

A experiência foi desenvolvida pela referida Universidade e pelo Sistema Municipal de Saúde de Parnaíba. Parnaíba é o segundo município mais populoso do estado do Piauí. A principal atividade econômica da cidade é a exportação de cera de carnaúba, óleo de babaçu, castanha de caju e couro. 0 agronegócio tem se desenvolvido na região devido ao Perímetro Irrigado dos Tabuleiros Litorâneos. Nos últimos 15 anos, a cidade tem se destacado por se constituir um centro de referência para a educação superior na região do Delta do Parnaíba.

0 processo teve início com a constituição de 
espaços de reflexão e contratualização técnica entre universidade e Sistema Municipal de Saúde de Parnaíba (SMSPHB) sobre a atuação da Psicologia na APS $1-5,8,11,12$, assim como o diálogo com a Coordenação de Educação Permanente em Saúde e a Superintendência de Atenção Básica de Saúde, além da inserção dos estudantes em Centros de Saúde da Família (CSF) que tinham maior potencial de acolhida de experiências de educação permanente em saúde. A atuação da Psicologia na APS é contratualizada com a SMSPHB e a equipe do CSF, considerando os pressupostos de atuação sistematizados pelo campo teórico; as resoluções do sistema do Conselho Federal de Psicologia - referência técnica para a atuação do Psicólogo na Atenção Básica em Saúde ${ }^{8}$; e a base jurídico-normativa do Ministério da Saúde ${ }^{15}$. Tais pressupostos são dialogados com a equipe do CSF, levando em conta sua realidade local e sua demanda no campo da saúde mental na APS.

A partir de tal processo, desenha-se um plano de trabalho de atuação da Psicologia na ABS, que é construído em permanente movimento, uma vez que a atuação na área não se dá de forma linear ou descrita por protocolos rígidos de atuação, mas tentando agir segundo as diretrizes da educação permanente em saúde, a qual compreende a relevância da horizontalidade e da atuação combinada entre universidade, serviço de saúde e comunidade. Para tanto, adotou-se um processo coletivo que parte da sistematização e da análise do diário de campo ${ }^{18,19}$ construídas por meio da identificação dos cenários de prática. A confluência desses cenários de formação possibilitou a indicação de caminhos para a atuação da Psicologia na APS.

A observação participante $e^{18,19}$ foi 0 eixo transversal do desenho metodológico deste artigo, pois proporcionou um espaço constante de reflexão e ação para a transformação da realidade, viabilizando a construção de espaços de descrição, análise, reflexão, problematização, facilitação psicossocial e sínteses da atuação da Psicologia na APS 1,35,8,11,12. 0 processo de discussão teórica, o plano de trabalho e o espaço de facilitação psicossocial dos estudantes foram gestando os cenários de atuação junto à equipe do CSF e à comunidade, além de levarem à construção de uma comunidade de prática - pressuposto de trabalho compartilhado das abordagens metodológicas participativas ${ }^{18}$.

0 diário de campo $^{18}$ emergiu dessa vivência como uma potencialidade desta metodologia a abordagem participativa - na medida em que todos os atores (universidade, serviço de saúde e comunidade) foram sujeitos de construção, além dos espaços de aprendizagem transversal na vivência, fortalecendo a articulação teórico-prática. 0 desafio metodológico posto foi a ausência de compreensão das metodologias participativas de atuação. As autoras e o autor deste trabalho são sujeitos epistêmicos e implicados do estudo na medida em que foram docentes e estudantes que vivenciaram a experiência, fortalecendo a articulação teóricoprática.

\section{RESULTADOS E DISCUSSÃO}

0 processo de inserção para a vivência em Psicologia na APS ${ }^{1,3-5,8,11-13}$ se deu por meio do estágio profissional curricular de caráter obrigatório do curso de graduação em Psicologia, tendo como ênfase curricular e marcador ético-político a Saúde Coletiva9. A atuação é orientada para o fazer da Psicologia na ABS por meio da estratégia do NASF ${ }^{1}$. Vale destacar que a localização do estágio da área de Psicologia na Saúde Coletiva $a^{7-9,11,12}$ aponta a importância de atuar na organização de sistemas e serviços, práticas de atenção à saúde e práticas sociais de abordagem comunitária, superando a atuação fundamentada nos pressupostos da clínica tradicional ${ }^{1,3-5,8,11-13}$ e da saúde pública tradicional 9 .

Inicialmente, começou-se a caminhada por meio da pactuação com a Secretaria Municipal de Saúde, a Superintendência de Atenção Básica em Saúde e a Coordenação da Unidade Básica de Saúde (UBS), orientados pelos pressupostos da Política Nacional de Educação Permanente em Saúde (PNEPS) ${ }^{8,17}$. Apresentou-se a esses atores institucionais o que se aponta na literatura sobre a Psicologia na Saúde Coletiva, as orientações do CFP e as diretrizes do MS em relação à atuação da Psicologia na $\mathrm{APS}^{1,3,5,8,11,12,16}$. Identificou-se, a partir desse processo, a necessidade de atuação do serviço de saúde e da comunidade. Desse processo, foi construído o plano de trabalho. Assim, compreendeu-se que a construção se deu coletivamente, de forma dialógica, uma relação entre universidade, sistema de saúde e comunidade.

Os cenários de atuação foram os CSF definidas pela Secretaria Municipal de Saúde. 0 critério de definição foi a abertura da gestão e dos trabalhadores aos processos de educação permanente em saúde ${ }^{17}$. Emergiram como cenários de atuação $0^{1-5,8,11-13}$ : a) territorialização ${ }^{13,14}$; b) clínica ampliada ${ }^{16}$; c) gestão da clínica; d) manejo de transtornos 
mentais comuns; e) promoção da saúde; f) grupos ${ }^{16}$; g) intersetorialidade; e h) fortalecimento da participação popular.

Iniciou-se com a territorialização ${ }^{13,14}$, o primeiro momento de inserção comunitária de levantamento de fontes primárias e secundárias ${ }^{18}$ acerca da comunidade, buscando compreendê-la histórica, social, econômica, cultural e sanitariamente, além de construir um vínculo com o território e a comunidade. A territorialização proporcionou a identificação e a atuação com o processo de saúde da comunidade, seu modo de vida, as principais instituições ali presentes, os principais atores e as potencialidades de cuidado no território. Essa é a ferramenta de partida no contexto da APS, pois fornece subsídios para uma atuação coerente com as necessidades e os anseios do território $0^{13,14}$. A partir dessa atuação, duas frentes integradas têm início: a) a clínica ampliada; e b) a abordagem comunitária.

No âmbito da clínica ampliada ${ }^{16}$, foram desenvolvidas estratégias de gestão da clínica, quais sejam: a) acolhimento; b) matriciamento; e c) projeto terapêutico singular. Conhecidas as demandas trazidas pela comunidade e contando com o apoio das instituições presentes, percebeuse a necessidade de trabalhar com o manejo de transtornos mentais comuns, manejo do uso abusivo de álcool e outras drogas, violação de direitos de crianças e adolescentes, problemas de desenvolvimento infantil, escuta psicossocial de usuários pertencentes a grupos minoritários (como mulheres, população LGBTQIA+ e população negra) e casos que envolvem suicídio. Assim, foram feitas sessões de escuta psicossocial para o manejo dos casos e a organização de estratégias de articulação com outros pontos de atenção à saúde mental na Rede de Atenção Psicossocial, como os Centros de Atenção Psicossocial (CAPS). Também emergiram dessa articulação de rede o acolhimento, o matriciamento, a construção de projetos terapêuticos singulares e os atendimentos individuais e domiciliares.

Além dos cenários de atuação descritos, por meio da articulação saúde-educação foram realizadas intervenções na escola da área de abrangência do CSF e construiu-se um espaço de escuta psicossocial para os professores e os estudantes. Emergiram questões como demandas em torno do desenvolvimento psicossocial de crianças e adolescentes, problemas de aprendizagem, situações de violação dos direitos da criança e do adolescente, ansiedade, casos de acolhimento à demanda de suicídio, entre outras.
As atividades desenvolvidas foram elaboradas coletivamente, envolvendo todos os atores implicados nesse processo e apontando para a compreensão da articulação intersetorial como uma importante ferramenta na produção de saúde.

No campo das abordagens comunitárias, por meio da construção coletiva da equipe da APS, iniciou-se o trabalho com o grupo, fortalecendo o processo de participação social e as articulações intersetoriais. 0 trabalho com grupos ${ }^{16}$ é mais um dos possiveis cenários de atuação, pois são espaços coletivos e constituem momentos para compartilhar e facilitar os processos de cuidado, podendo ser pedagógicos, operativos e terapêuticos, permitindo a atuação coletiva e de integração da clínica à Saúde Coletiva. Surgiram, no território, demandas de grupos de educação em saúde para o manejo de algumas questões, como grupo de acolhimento a transtornos mentais comuns, violência de gênero, saúde da mulher e outras propostas vinculadas às ações programáticas e/ou às campanhas de saúde.

Considerando o campo da atuação intersetorial, identificaram-se demandas de atuação integradas a outras instituições e políticas sociais, como educação, por meio da demanda de cuidado a crianças em situação de violência; diálogos com o Conselho Tutelar na atuação contra a violação dos direitos da criança e do adolescente; diálogo com a Assistência Social, uma vez que muitas atuações dialogam com benefícios sociais e outros direitos sociais; articulação com o Conselho Municipal de Saúde; e visitas técnicas a órgãos que atuam na abordagem da violência de gênero, dentre outras.

Levando em conta o campo da participação popular em saúde, o psicólogo na APS desempenha o papel de apoiador, produzindo análises e intervenções que visem à transformação dos processos de implicação política dos sujeitos.

Deste estudo, emergiram espaços de fortalecimento da participação popular em saúde por meio da articulação entre a participação popular institucionalizada e não institucionalizada. Como espaço desta última, adveio o diálogo com movimentos sociais em saúde, como as associações comunitárias, as pastorais sociais e os movimentos que atuam na defesa da diversidade sexual e da comunidade LGBTQIA+, além do movimento Hip Hop de arte e cultura, entre outros. Como espaços de apoio de participação popular institucionalizada, surgiram a participação no Conselho Municipal de Saúde de Parnaíba, o apoio na organização de Conselhos 
Municipais de Saúde e as conferências de saúde. A atuação nesse cenário consistia em fortalecer a rede de apoio social no cuidado, desenvolver abordagens de trabalho comunitário e fortalecer a cidadania por meio da facilitação de construção da implicação política dos sujeitos. Para a elaboração de micropolíticas de fomento à participação popular em saúde, mostram-se necessários processos de subjetivação que sustentem as plataformas de cuidado. Nesse sentido, o psicólogo, como qualquer outro profissional da APS, atua a fim de agenciar a formação dessas subjetividades coletivas no território. Cabe ressaltar que a inserção no território e seu desempenho estão diretamente ligados à abertura/ao acolhimento por parte das equipes e da gestão em relação aos estagiários e às suas respectivas propostas de trabalho, que podem ser ampliadas ou limitadas na mesma proporção.

Considerando os cenários de atuação descritos, a atuação da Psicologia na APS ${ }^{11-14,16}$ possibilitou 0 diálogo que emerge da territorializaçã $0^{13}$, permitindo uma compreensão profunda da determinação social da saúde ${ }^{14}$. Esse processo potencializa um diálogo entre a clínica ampliada ${ }^{16}$ e a Saúde Coletiva ${ }^{9}$, buscando uma atuação coerente com a organização de sistemas e serviços de saúde e com as necessidades sociais em saúde mental ${ }^{2,7}$, através do fortalecimento de práticas de abordagens comunitárias, participação popular em saúde e desenvolvimento da cidadania. Isto é, a atuação da Psicologia na Atenção Primária em Saúde ${ }^{1-5,7,11,12}$ tem um potencial de diálogo com o campo da Saúde Coletiva ${ }^{9}$, permitindo desenvolver experiência com o SUS e com as práticas sociais em saúde instituintes de produção de subjetividade que emergem dos movimentos de mulheres e feministas, comunidades LGBTQIA+, população quilombola, povos de terreiros, população de rua, entre outros. A experiência permitiu uma compreensão ampla sobre os sujeitos, processos de subjetivação e a realidade histórico-cultural como dimensões indissociáveis, apontando a importância do diálogo da clínica com a Saúde Coletiva $a^{2-5,7,11,12}$. Como também o diálogo entre a dimensão micropolítica e os processos macropolíticos de organização do Estado e da sociedade.

É importante colocar que, atualmente, este cenário de atuação da Psicologia na ABS tem sofrido ameaças importantes com o recuo das políticas de saúde na atual conjuntura política sanitária brasileira ${ }^{20-22}$. Nos últimos anos, o país tem sofrido transformações importantes na Política Nacional de Atenção Básica em Saúde $20-22$ com a mudança na reorientação no âmbito do financiamento e organização do processo de trabalho na Atenção Primária à Saúde, composição e financiamento das equipes de APS, na abordagem comunitária em saúde, impactando de forma consistente a atuação da Psicologia na APS no Brasil.

Como impactos da experiência compartilhados e sistematizados nos diários de campo por meio das oficinas participativas de avaliação do Estágio Profissional, apontaram-se as seguintes questões: a experiência permitiu o fortalecimento do diálogo entre universidade, serviço de saúde e comunidade - como dispositivo de educação permanente em saúde; articulação teórico-prática; aprofundou a formação do Psicólogo para o SUS na medida em que diversos egressos continuaram seus processos de formação e atuação em Programas de Residência Multiprofissional em Saúde. Além disso, destacamse também o processo de compreensão da atuação da Psicologia na Atenção Básica em Saúde e da Saúde Mental na ABS como dispositivo da RPB pelos trabalhadores e comunidade e, finalmente, as contribuições dos estagiários no fortalecimento da atuação no âmbito da saúde mental na Atenção Básica em Saúde em Parnaíba, município que tem enfrentado desafios importantes neste campo.

\section{CONCLUSÃO}

A Psicologia na ABS, como área de conhecimento e campo de práticas profissionais, surgiu do contexto de ampliação do direito à saúde por meio da Reforma Sanitária, da Reforma Psiquiátrica e da criação do SUS. Mostra-se necessário afirmar que o marco político-ideológico desse processo foi o movimento da Reforma Psiquiátrica, que contribuiu para a reorganização de um modelo de atenção à saúde mental que buscava superar o manicômio como cenário de cuidado, a organização da Rede de Atenção Psicossocial e a ampliação da atenção à saúde no âmbito do SUS.

Dessa forma, a partir da criação da estratégia do NASF, institucionalizou-se o processo de inserção da Psicologia na APS. É importante afirmar que, naquele momento de criação, havia desafios no cotidiano da formação e do trabalho no setor saúde que persistem até os dias de hoje.

Considerando tal processo, surge na academia um esforço teórico-prático de organização dos possíveis cenários de atuação. Em paralelo, outros atores, como o CFP e os Conselhos Regionais de 
Psicologia (CRPs), a Associação Brasileira de Ensino de Psicologia (ABEP), as secretarias municipais de saúde e o movimento estudantil, atuam para fortalecer a leitura, a análise, a implementação e a sistematização da atuação da Psicologia na APS. Assim, a organização deste relato de experiência é fruto de tal movimento de atores sociais em defesa da atuação da Psicologia em direção à ampliação do direito à saúde e às necessidades de saúde da população.

Compreende-se que há diversos desafios nesse processo, uma vez que é preciso fortalecer a educação permanente em saúde como espaço de diálogo entre universidade e sistemas de saúde. Encontraramse desafios tanto na ausência de compreensão da atuação da Psicologia na $A B S$, da saúde mental na $A B S$, quanto do campo da educação permanente em saúde.

Emergiram como cenários de atuação a territorialização $0^{13,14}$ a clínica ampliada, o matriciamento, o projeto terapêutico, os transtornos mentais comuns, o desenvolvimento psicossocial de crianças e adolescentes, a escuta psicossocial em situações de violência, o acolhimento e a articulação da Rede de Atenção Psicossocial para o manejo dos transtornos mentais severos, matriciamento, etc. Como estratégias de abordagens comunitárias, emergiram a territorialização ${ }^{13,14}$, o trabalho com grupos, a intersetorialidade e o fortalecimento da participação popular em saúde, entre outras.

Concluindo, compreende-se que o campo da Psicologia na APS se encontra em construção. Mostrase importante reafirmar que a Psicologia amplia sua área de conhecimento e seu espaço de atuação direcionando o trabalho ao campo da determinação social da saúde, bem como potencializando o diálogo da clínica com a Saúde Coletiva. Outro posicionamento ético-político é o diálogo com os pressupostos da Reforma Psiquiátrica Democrática Italiana, que fomenta a atuação integrada com as dimensões teórica, técnico-assistencial, jurídica, política e sociocultural.

Atualmente, tal proposição se encontra fortemente ameaçada pela agenda ultraneoliberal, conservadora, pautada por cortes de gastos, que atinge o SUS, além das mudanças na atual política nacional de APS. Estas últimas eliminam as ações de clínica ampliada, de abordagens comunitárias, de atuação do NASF e dos agentes comunitários de saúde (ACS). Entende-se que essas ações consistem em recuos importantes considerando os avanços do
SUS, a gestão em saúde, a ampliação da atenção à saúde e a própria organização do setor saúde no país.

\section{CONTRIBUIÇÃO DOS AUTORES}

\author{
Ana Ester Maria Melo Moreira, Brenda Brito \\ Ramos, Thalia Ariadne Peña Aragão, Letícia \\ Dias Baroni, Kerolayne Castro Fontenele e José \\ Lucas Soares Araújo contribuíram integralmente \\ no processo de formulação das experiências, nas \\ atividades de campo, no processo de delineamento, \\ elaboração, sistematização e revisão crítica do \\ artigo.
}

\section{REFERÊNCIAS}

1. Brasil. Portaria n. ${ }^{0}$ 154, de 24 de janeiro de 2008. Cria os Núcleos de Apoio à Saúde da Família - NASF. Diário Oficial [da] República Federativa do Brasil. 2008 jan. 24; Seção 1. p. 47-50.

2. Amarante PDC. Saúde, desinstitucionalização e novas estratégias de cuidado. In: Giovanella L, Escorel S, Lobato LVC, Noronha JC, Carvalho AI, organizadores. Políticas e sistemas de saúde no Brasil. Rio de Janeiro: Ed. Fiocruz; 2008. p. 735-60.

3. Polejack L, Totugui LM, Gomes MGP, Conceição GMI. Atuação do psicólogo nas políticas públicas de saúde: caminhos, desafios e possibilidades. In: Polejack L, Vaz AMA, Gomes PMG, Wichrowski VC, organizadores. Psicologia e políticas públicas na saúde: experiências, reflexões, interfaces e desafios. Porto Alegre: Rede Unida; 2015. p. 31-50.

4. Iglesias A, Belotti M, Avellar, LZ. Análise documental sobre as normativas do trabalho no Núcleo Ampliado de Saúde da Família. Psicol Ciênc Prof [serial on the internet]. 2019 [cited 2020 May 28]; 39 (1):1-14. Available from: https://www.scielo. $\mathrm{br} / \mathrm{pdf} / \mathrm{pcp} / \mathrm{v39} / 1982-3703-\mathrm{pcp}-39$-e185025.pdf

5. Polejack L, Gomes PMG, Miranda MRN, Machado MPM. A psicologia na atenção básica: descobertas, conquistas, considerações e desafios no NASF. In: Polejack L, Vaz AMA, Gomes PMG, Wichrowski VC, organizadores. Psicologia e políticas públicas na saúde: experiências, reflexões, interfaces e desafios. Porto Alegre: Rede Unida; 2015. p. 99-127.

6. Macerata I, Soares JGN, Ramos JFC. Apoio como cuidado de territórios existenciais: atenção básica e a rua. Interface (Botucatu) [serial on the internet]. 2014 [cited 2020 May 28]; 18(Suppl l):919-30. Available from: https://www.scielo.br/pdf/icse/ v18s1/1807-5762-icse-18-1-0919.pdf

7. Macedo JPS, Soares FBP. Interseções entre a psicologia da saúde e saúde coletiva: uma revisão 
integrativa. Rev Psicol Saúde [serial on the internet]. 2020 [cited 2020 May 28]; 12(1):33-47. Available from: http://pepsic.bvsalud.org/pdf/ rpsaude/v12n1/v12n1a03.pdf

8. Conselho Federal de Psicologia. Referências técnicas para atuação do Psicólogos(as) na Atenção Básica em Saúde. Brasília (DF): Conselhos Regionais de Psicologia e Centro de Referência Técnica em Psicologia e Políticas Públicas; 2019.

9. Paim JS, Almeida Filho N. Saúde coletiva como campo de saberes e práticas. In: Paim JS, Almeida Filho N. Saúde coletiva: teoria e prática. Rio de Janeiro: MedBook; 2014. p. 3-12.

10. Giovanella L. Atenção primária em saúde. In: Giovanella L, Escorel S, Lobato LVC, Noronha JC, Carvalho AI, organizadores. Políticas e sistemas de saúde no Brasil. Rio de Janeiro: Ed. Fiocruz; 2008. p. 575-626.

11. Nepomuceno LB, Pontes RJS. 0 espaço socioprofissional da Estratégia de Saúde da Família sob a perspectiva de psicólogos. Psicol Ciência Prof [serial on the internet]. 2017 [cited 2020 May 28]; 37(2):289-303. Available from: https://www.scielo. $\underline{\mathrm{br} / \mathrm{pdf} / \mathrm{pcp} / \mathrm{v} 37 \mathrm{n} 2 / 1982-3703-p c p-37-2-0289 . p d f}$

12. Nepomuceno LB, Brandão IR. Psicólogos na Estratégia Saúde da Família: caminhos percorridos e desafios a superar. Psicol Ciênc Prof [serial on the internet]. 2011 [cited 2020 May 28]; 31(4):76277. Available from: https://www.scielo.br/pdf/pcp/ v31n4/v31n4a08.pdf

13. Pessoa VM, Rigotto RM, Carneiro FF, Teixeira ACA. Sentidos e métodos de territorialização na atenção primária à saúde. Ciênc saúde colet [serial on the internet]. 2013 [cited 2020 May 28]; 18(8):225362. Available from: https://www.scielo.br/pdf/csc/ v18n8/09.pdf

14. Ximenes VM, Lemos EC, Silva AMS, Abreu MKA, Esmeraldo Filho CE, Gomes LM. Saúde comunitária e psicologia comunitária: suas contribuições às metodologias participativas. Psicol Pesq [serial on the internet]. 2017 [cited 2020 May 28]; 2(2):413. Available from: http://pepsic.bvsalud.org/pdf/ psipesq/v11n2/02.pdf

15. Brasília. Ministério da Saúde. Departamento de Atenção Básica. Departamento de Ações Programáticas Estratégicas. Cad Atenção Básica. 2013; (34).

16. Cunha GT. A construção da clínica ampliada na atenção básica. São Paulo: Hucitec; 2005.

17. Ceccim RB. Emergência de um “campo de ação estratégica": ordenamento da formação e educação permanente em saúde. Sanare (Sobral,
Online) [serial on the internet]. 2019 [cited 2020 May 28]; 18(1):68-80. Available from: https:// sanare.emnuvens.com.br/sanare/article/view/ $\underline{1307 / 688}$

18. Montero M. Hacer para transformar: el método de la psicología comunitaria. Buenos Aires: Paidós; 2006.

19. Fernandes MBF, Moreira MR. Considerações metodológicas sobre as possibilidades de aplicação da técnica de observação participante na saúde coletiva. Physis [serial on the internet]. 2013 [cited 2020 May 28]; 23(2):511-29. Available from: $\quad$ https://www.scielo.br/scielo.php ? pid =S01033312013000200010\&script=sci abstract $\& t \operatorname{lng}=p t$

20. Paim JS. A COVID-19, a atualidade da reforma sanitária e as possibilidades do SUS. In Santos AO, Lopes LT, organizadores. Reflexões e futuro. Brasília (DF): Conselho Nacional de Secretários de Saúde; 2021. p. 310-20.

21. Giovanella L, Franco CM, Almeida PF. Política Nacional de Atenção Básica: para onde vamos? Ciênc saúde colet. $2020 ; 25(4): 1475-81$.

22. Morosini MVG, Fonseca AF, Baptista TWF. Previne Brasil, Agência de Desenvolvimento da Atenção Primária e Carteira de Serviços: radicalização da política de privatização da atenção básica? Cad. saúde pública. 2020; 36(9):e00040220.

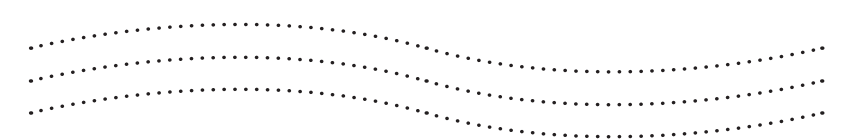

